\title{
Laser Light Scattering Study of the Degradation of Poly(sebacic anhydride) Nanoparticles
}

\author{
JIE FU, ${ }^{1,2} \mathrm{CHI} \mathrm{WU}^{1,3}$ \\ ${ }^{1}$ Open Laboratory of Bond-Selective Chemistry, Department of Chemical Physics, University of Science and Technology \\ of China, Hefei, Anhui, China \\ ${ }^{2}$ College of Material Science and Engineering, Wuhan University of Technology, Wuhan, Hubei, China \\ ${ }^{3}$ Department of Chemistry, Chinese University of Hong Kong, Shatin, N.T., Hong Kong
}

Received 28 October 1999; revised 31 October 2000; accepted 20 December 2000

\begin{abstract}
Poly(sebacic anhydride) (PSA) is biocompatible and degradable in basic media. We micronized this water-insoluble polymer into stable polymeric nanoparticles via a microphase inversion. Such PSA nanoparticles degraded much faster than bulk PSA. The influence of the surfactant, temperature, and $\mathrm{pH}$ on the degradation of the PSA nanoparticles was investigated by a combination of static and dynamic laser light scattering. Under each condition, the degradation rate was nearly constant up to a $75 \%$ weight loss; that is, the degradation was close to zero-order. The degradation rate increased with the $\mathrm{pH}$ and temperature. Biomedical applications of such PSA nanoparticles are suggested. ( 2001 John Wiley \& Sons, Inc. J Polym Sci B: Polym Phys 39: 703-708, 2001
\end{abstract}

Keywords: poly(sebacic anhydride); nanoparticle; biodegradation; laser light scattering

\section{INTRODUCTION}

Synthetic degradable polymers are attractive and important new materials because of their environmentally friendly nature and biomedical applications. For example, some of them can be used for surgical sutures, orthopedic implants, scaffolds for cells in tissue engineering, and controllable drug release depots. ${ }^{1-6}$ The degradation of a polymer backbone chain can be enzymatic or hydrolytic. In general, hydrolytic degradation is preferred for biomedical applications because it is less dependent on the implantation site and the patient. ${ }^{7}$ Hydrolytic degradation can occur either in the bulk or on the surface of a polymer. ${ }^{8}$ Cur-

Correspondence to: C. Wu, Department of Chemistry, Chinese University of Hong Kong, Shatin, N.T., Hong Kong (Email: chiwu@cuhk.edu.hk)

Journal of Polymer Science: Part B: Polymer Physics, Vol. 39, 703-708 (2001) (C) 2001 John Wiley \& Sons, Inc. rently, most available biodegradable polymers, such as polylactide and polycaprolactone, have the characteristics of bulk erosion; that is, water penetrates into the polymer and degrades it internally and externally. However, the surface erosion has the advantage of zero-order kinetics, that is, a constant release rate, if there is nearly no change in the total surface area during the erosion.

It is known that the degradation of polyanhydrides is mainly on the surface. ${ }^{9-12}$ They are potential biomedical materials. Each anhydride bond $(\mathrm{CO}-\mathrm{O}-\mathrm{CO})$ on the backbone chain can hydrolytically break down into two carboxylic acids, so that an initially insoluble long polymer chain can be cleaved into shorter and soluble fragments. Tissue reactions and toxicological studies showed that polyanhydrides were biocompatible. ${ }^{13,14}$ For example, they are currently used in clinics to deliver BCNU (carmustine, 1,3-bis[2- 
chloroethyl]-1-nitro-sourea) locally within the brain to treat patients with brain tumors. ${ }^{15}$ However, it is difficult, if not impossible, to keep the surface area of a bulk polyanhydride sample constant during the erosion except for a very thin film. Our previous studies revealed that if a polymer is micronized into small particles with a diameter of a few hundred nanometers, the degradation of each nanoparticle is very fast under proper conditions. The decrease in the number of particles is linear in the degradation time. Our results indicated that instead of keeping a constant total surface area, we could use polymeric nanoparticles to achieve zero-order degradation kinetics. Using polymeric nanoparticles as drug carriers can increase the delivery efficiency, reduce associated adverse effects, and make injection possible. ${ }^{16-28}$ In this study, we synthesized poly(sebacic acid) (PSA), micronized it into nanoparticles, and investigated its degradation kinetics with laser light scattering (LLS). This is a fundamental portion of a large project related to biomedical applications.

\section{EXPERIMENTAL}

\section{Sample Preparation}

Sebacic acid was recrystallized three times from ethanol. Acetic anhydride was purified by distillation. Tetrahydrofuran (THF) and chloroform were refluxed and distilled over calcium hydride. Toluene and $n$-hexane were predried with 4 - $\AA$ molecular sieves and distilled. Sodium laurylsulfate (SDS), polyoxyethylene20 sorbitan monolaurate (Tween20), polyoxyethylene20 sorbitan monostearate (Tween60), sorbitan monolaurate (Span20), and other reagents were used as received without further purification. The synthesis of poly(sebacic anhydride) (PSA) via melting polycondensation was as follows. ${ }^{29-31}$ Refluxing recrystallized sebacic acid in acetic anhydrides resulted in a mixture of anhydride oligomers. The resultant oligomers were recrystallized from toluene and washed with $n$-hexane. The polymerization of these oligomers was conducted in a glass tube with a volume of approximately $35 \mathrm{~cm}^{3}$ with a nitrogen inlet at $180{ }^{\circ} \mathrm{C}$ under $0.01 \mathrm{mmHg}$. Acetic anhydride was removed under vacuum and collected in a liquid nitrogen trap. The resultant PSA was purified by precipitation in dry $n$-hexane from a chloroform solution. The average molar mass of PSA used in this study was $4.72 \times 10^{4} \mathrm{~g}$ $\mathrm{mol}^{-1}$, as measured by LLS.

\section{Micronization of PSA}

The PSA nanoparticles were prepared by the addition of a dilute PSA THF solution dropwise into an excess of water containing surfactant under constant stirring. As expected, after each drop of the THF solution was added, THF quickly diffused and mixed with water, and the insoluble hydrophobic PSA chains collapsed and aggregated in water to form small PSA nanoparticles stabilized by the surfactant molecules. The THF was removed under reduced pressure. Hereafter, the PSA nanoparticles stabilized by SDS, Tween20, Tween60, and Span20 are denoted SDS-PSA, Tween20-PSA, Tween60-PSA, and Span20-PSA, respectively.

\section{LLS}

A modified commercial LLS spectrometer (ALV/ SP-125) equipped with an ALV-5000 multi- $\tau$ digital time correlator and a solid-state laser (ADLAS DPY425II; outpower $=\sim 400 \mathrm{mV}$ at $\lambda=532$ $\mathrm{nm}$ ) was used. In static LLS, the angular dependence of the excess absolute time-average scattered intensity, that is, the Rayleigh ratio $R_{v v}(q)$, of a dilute dispersion leads to the weight-average molar mass $M_{w}$, the second virial coefficient $A_{2}$, and the root-mean-square $z$-average radius $\left\langle R_{g}{ }^{2}\right\rangle_{z}{ }^{1 / 2}$ (or $\left.\left\langle R_{g}\right\rangle\right)$, ${ }^{32}$ where $q$ is the scattering vector. In dynamic LLS, the Laplace inversion of a measured intensity-intensity time correlation function $G^{(2)}(t, q)$ in the self-beating mode results in a line-width distribution $G(\Gamma) .{ }^{32,33}$ For pure diffusive relaxation, $\left(\Gamma / q^{2}\right)_{q \rightarrow 0, c \rightarrow 0}$ leads to the translational diffusion coefficient $D$ or the hydrodynamic radius $R_{h}$ via the Stokes-Einstein equation: $R_{h}=k_{B} T /(6 \pi \eta D)$, where $k_{B}, T$, and $\eta$ are the Boltzmann constant, absolute temperature, and solvent viscosity, respectively. Therefore, $G(\Gamma)$ can be converted to a distribution of the hydrodynamic radius $\left[f\left(R_{h}\right)\right]$. The details of LLS theory and instrumentation can be found elsewhere. ${ }^{32,33}$

\section{Degradation}

Buffer solutions were used to control $\mathrm{pH}$ in the degradation of PSA. The $\mathrm{OH}^{-}$group acted as a catalyst in the degradation. The PSA dispersion and buffer solutions used in LLS were clarified by 0.8- and 0.1- $\mu \mathrm{m}$ Millipore filters, respectively, to remove dust. In a typical degradation experiment, a proper amount of the resultant dust-free PSA nanoparticle dispersion was added in situ into a 2-mL dust-free buffer solution. Both $R_{v v}(q)$ and 


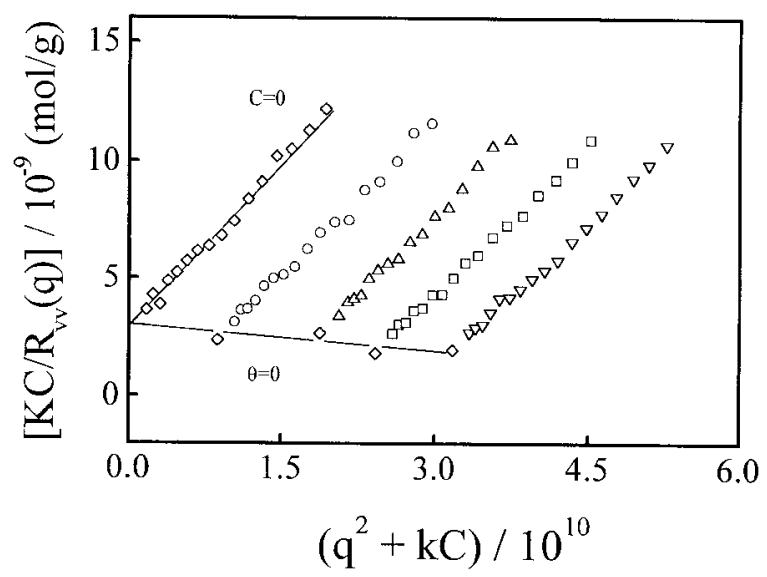

Figure 1. Typical Zimm plot of SDS-PSA nanoparticles in deionized water at $25^{\circ} \mathrm{C}$, where $C$ ranges from $4.27 \times 10^{-6}$ to $1.06 \times 10^{-5} \mathrm{~g} / \mathrm{mL}$.

$G^{(2)}(t, q)$ were simultaneously measured during the degradation.

\section{RESULTS AND DISCUSSION}

Figure 1 shows a typical Zimm plot of the SDS-PSA nanoparticles in water at $25^{\circ} \mathrm{C}$. The extrapolation of $\left[K C / R_{v v}(q)\right]_{C \rightarrow 0, q \rightarrow 0}$ leads to $M_{w}$, and the slopes of $\left[K C / R_{v v}(q)\right]_{C \rightarrow 0}$ versus $q^{2}$ and $\left[K C / R_{v v}(q)\right]_{q \rightarrow 0}$ versus $C$, respectively, lead to $\left\langle R_{g}\right\rangle$ and $A_{2}$, where $K$ is a constant for a given dispersion and temperature. Table I summarizes the values of $M_{w},\left\langle R_{g}\right\rangle$, and $A_{2}$ for four different kinds of PSA nanoparticles, where the average particle density $\langle\rho\rangle$ was estimated from $M_{w}$ and $\left\langle R_{h}\right\rangle$ by $\langle\rho\rangle=M_{w} N_{A} /\left[(4 / 3) \pi\left\langle R_{h}\right\rangle^{3}\right]$. The values of $\langle\rho\rangle$ are much lower than for the bulk polymer $\left(\sim 1 \mathrm{~g} / \mathrm{cm}^{3}\right)$, indicating that the PSA nanoparticles are made of loosely aggregated chains accompanied by a lot of water. The positive values of $A_{2}$ reveal that Tween 20 and Tween 60 are better stabilizers. This could be attributed to a more complete coverage of these two kinds of stabilizers on the particle surface.

Figure 2 shows that the PSA nanoparticles in water at $25{ }^{\circ} \mathrm{C}$ are narrowly distributed. For each



Figure 2. Typical hydrodynamic radius distributions $f\left(R_{h}\right)$ of SDS-PSA, Tween20-PSA, Tween60-PSA, and Span20-PSA nanoparticles in deionized water at $25^{\circ} \mathrm{C}$.

$f\left(R_{h}\right)$, an average hydrodynamic radius was calculated by $\int_{0}^{\infty} f\left(R_{h}\right) R_{h} d R_{h}$. The values of $\left\langle R_{h}\right\rangle$ for the SDS-PSA, Tween20-PSA, Tween60-PSA, and Span20-PSA nanoparticles are also listed in Table I. It is known that the $\left\langle R_{g}\right\rangle\left\langle\left\langle R_{h}\right\rangle\right.$ ratio reflects the conformation of a polymer chain or the density distribution of a colloid particle. For example, for a flexible coil chain in a good solvent, $\left\langle R_{g}\right\rangle /\left\langle R_{h}\right\rangle$ $\sim 1.5$; for a uniform nondraining sphere, $\left\langle R_{g}\right\rangle /\left\langle R_{h}\right\rangle$ $=0.774$. The values of $\left\langle R_{g}\right\rangle /\left\langle R_{h}\right\rangle \sim 1.1-1.2$ in Table I also suggest that the nanoparticles are made of loosely aggregated PSA chains, ${ }^{34}$ which agrees well with lower values of $\langle\rho\rangle$. Figure 2 also shows that the particles formed in the presence of SDS are much more narrowly distributed. This is because SDS can form uniform micelles in water before the addition of the polymer THF solution so that the hydrophobic polymer chains are dispersed inside these micelles.

Figure 3 shows that the decrease of $\left[R_{v v}(q)\right]_{t} /$ $\left[R_{v v}(q)\right]_{o}$ is nearly linear in the degradation time. It is known that $R_{v v}(q) \propto C M_{w}$ at $C \rightarrow 0$ and $q \rightarrow$ 0 . Therefore, the decrease of $\left[R_{v v}(q)\right]_{t} /\left[R_{v v}(q)\right]_{o}$ can be related to the decrease of either $M_{w}$ or $C$ or both. However, dynamic LLS results showed that there was no change in the size of the nanopar-

Table I. Laser Light Scattering Characterization of the PSA Nanoparticles in Deionized Water at $25^{\circ} \mathrm{C}$

\begin{tabular}{lcccccc}
\hline Nanoparticles & $M_{w}(\mathrm{~g} / \mathrm{mol})$ & $A_{2}\left(\mathrm{~mol} \cdot \mathrm{cm}^{3} / \mathrm{g}^{2}\right)$ & $\left\langle R_{g}\right\rangle(\mathrm{nm})$ & $\left\langle R_{h}\right\rangle(\mathrm{nm})$ & $\left\langle R_{g}\right\rangle /\left\langle R_{h}\right\rangle$ & $\langle\rho\rangle\left(\mathrm{g} / \mathrm{cm}^{3}\right)$ \\
\hline SDS-PSA & $3.34 \times 10^{8}$ & $-5.217 \times 10^{-5}$ & 216 & 186 & 1.16 & 0.021 \\
Tween20-PSA & $3.72 \times 10^{8}$ & $1.117 \times 10^{-4}$ & 206 & 180 & 1.14 & 0.025 \\
Tween60-PSA & $3.14 \times 10^{8}$ & $3.326 \times 10^{-4}$ & 181 & 162 & 1.11 & 0.017 \\
Span20-PSA & $2.32 \times 10^{8}$ & $-2.319 \times 10^{-5}$ & 198 & 185 & 1.07 & 0.015 \\
\hline
\end{tabular}




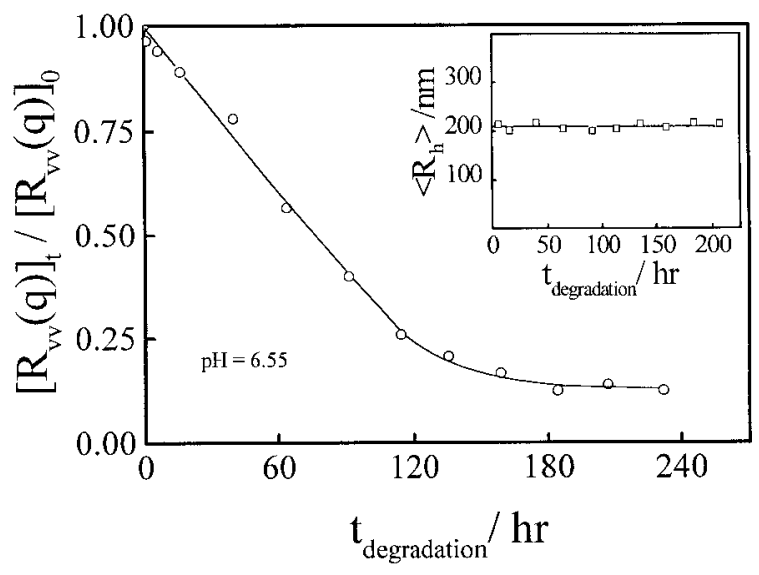

Figure 3. Degradation time dependence of $\left[R_{v v}(q)\right]_{t} /$ $\left.R_{v v}(q)\right]_{0}$ for SDS-PSA nanoparticles in deionized water at $25^{\circ} \mathrm{C}$, where $C_{o}$ is $3.88 \times 10^{-6} \mathrm{~g} / \mathrm{mL}$. The inset shows a corresponding time dependence of the average hydrodynamic radius $\left\langle R_{h}\right\rangle$.

ticles during the degradation (see the inset in Fig. 3 ), that is, no change in $M_{w}$, but the gravimetrical method confirmed the degradation of the particles. Apparently, this is a contradiction. In principle, the degradation should lead to a decrease in the particle size. The constant particle size reveals that the degradation of each particle was so fast that LLS could only detect the remaining nondegraded PSA nanoparticles, not those low molar mass degradation products. The decrease of $\left[R_{v v}(q)\right]_{t} /\left[R_{v v}(q)\right]_{o}$ actually reflected the decrease of the nanoparticle number, that is, the decrease of the relative concentration $\left(C_{t} / C_{o}\right)$. Figure 3 reveals that up to a $75 \%$ weight loss, the degradation is close to zero-order, but the degradation is fairly slow at $\mathrm{pH} 6.55$.

Figure 4 shows the $\mathrm{pH}$ dependence of the degradation of the SDS-PSA nanoparticles at $25^{\circ} \mathrm{C}$. In comparison with Figure 3 , the degradation rate at $\mathrm{pH} 11.0$ is approximately 100 times faster than at $\mathrm{pH}$ 6.5. For comparison, we also studied the degradation of a PSA disk with a diameter of 5 $\mathrm{mm}$ and a thickness of $2 \mathrm{~mm}$. Figure 5 shows that the disk degraded much more slowly than the nanoparticles for a given $\mathrm{pH}$ and temperature, which can be attributed to the difference in the surface area between the nanoparticles and the disk. It has been found that the degradation rate increases with the surface area of the disk. Our results clearly demonstrate that a combination of LLS and micronization provides a fast and accurate method to evaluate the degradation of PSA.

Figure 6 indicates that a surfactant as a stabilizer can also affect the degradation rate of the

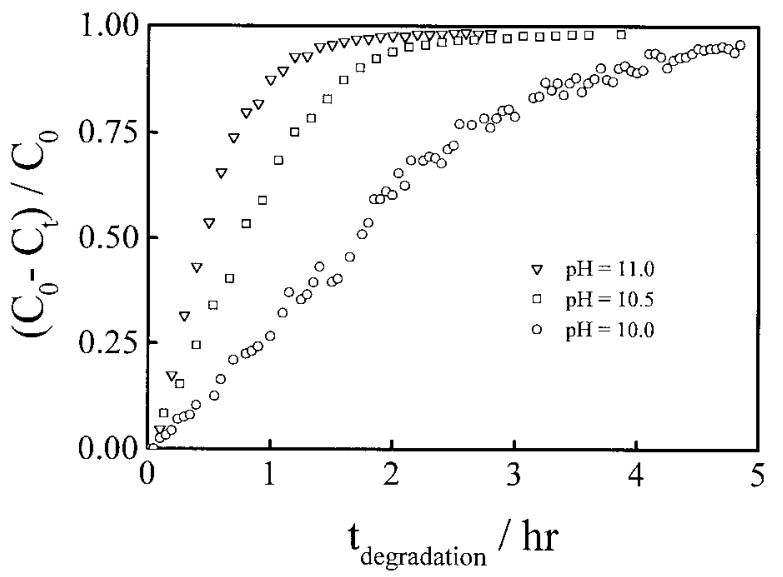

Figure 4. $\mathrm{pH}$ dependence of the degradation of SDSPSA nanoparticles at $25{ }^{\circ} \mathrm{C}$, where $C_{o}$ is $3.88 \times 10^{-6}$ $\mathrm{g} / \mathrm{mL}$ and $C_{t}$ is the PSA nanoparticle concentration at time $t$.

PSA nanoparticles in an aqueous solution. According to the decreasing order of the degradation rate, Span20-PSA is greater than Tween20-PSA, and Tween60-PSA is greater than SDS-PSA. Relatively, the slowest degradation of SDS-PSA can be attributed to the fact that SDS is anionic, slowing the attack of anionic $\mathrm{OH}^{-}$ions on the anhydride bond. For nonionic surfactants, Span20-PSA degrades faster than Tween20-PSA and Tween60-PSA because the steric barrier of the polyoxyethylene chains of Tween 20 and Tween60 introduce steric barriers and hinder the $\mathrm{OH}^{-}$ions from attacking the anhydride bond.

Figure 7 shows that for SDS-PSA, the degradation rate increases with the temperature. In each case, the initial degradation is nearly a lin-

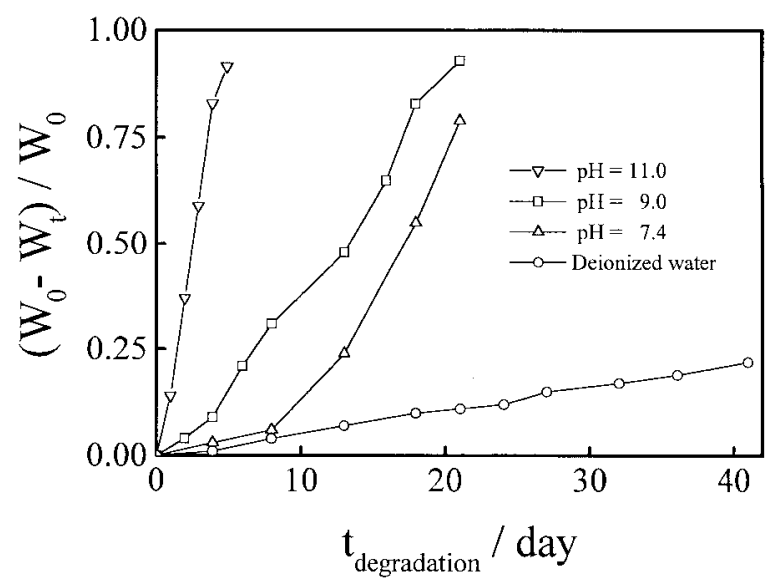

Figure 5. $\mathrm{pH}$ dependence of the degradation of a compression-molded PSA disk with a diameter of $5 \mathrm{~mm}$ and a thickness of $2 \mathrm{~mm}$ at $25^{\circ} \mathrm{C}$. 


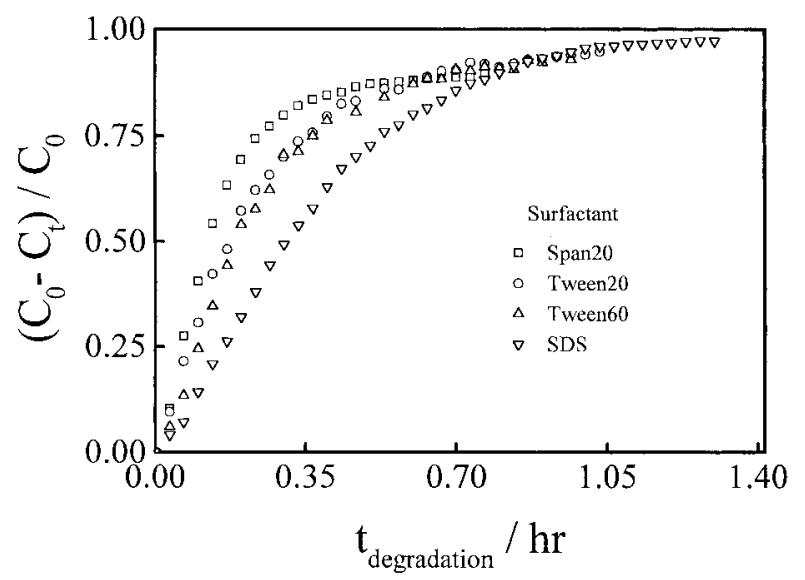

Figure 6. Surfactant dependence of the degradation of PSA nanoparticles at $37^{\circ} \mathrm{C}$, where $C_{o}$ is $3.88 \times 10^{-6}$ $\mathrm{g} / \mathrm{mL}$ and the $\mathrm{pH}$ is 10.5 .

ear function of the degradation time. The leastsquare fitting of the initial linear portion of each " $\left(C_{0}-C_{t}\right) / C_{0}$ versus $t$ " leads to an initial degradation rate $v$, defined as $\lim _{t \rightarrow 0}\left\{\left[\left(C_{0}-C_{t}\right) / C_{0}\right] t\right\}$. Figure 8 shows typical Arrhenius plots for the degradation of the PSA nanoparticles stabilized with different surfactants. The activation energies of the SDS-PSA, Tween20-PSA, Tween60PSA, and Span20-PSA degradation are 43, 56, 52, and $88 \mathrm{~kJ} / \mathrm{mol}$, respectively. Although the activation energy for SDS-PSA is the smallest, electrostatic repulsion between $\mathrm{OH}^{-}$and SDS makes the collision frequency low, so the degradation rate of SDS-PSA is still the slowest.

\section{CONCLUSION}

The micronization of water-insoluble PSA into a stable nanoparticle dispersion not only makes its

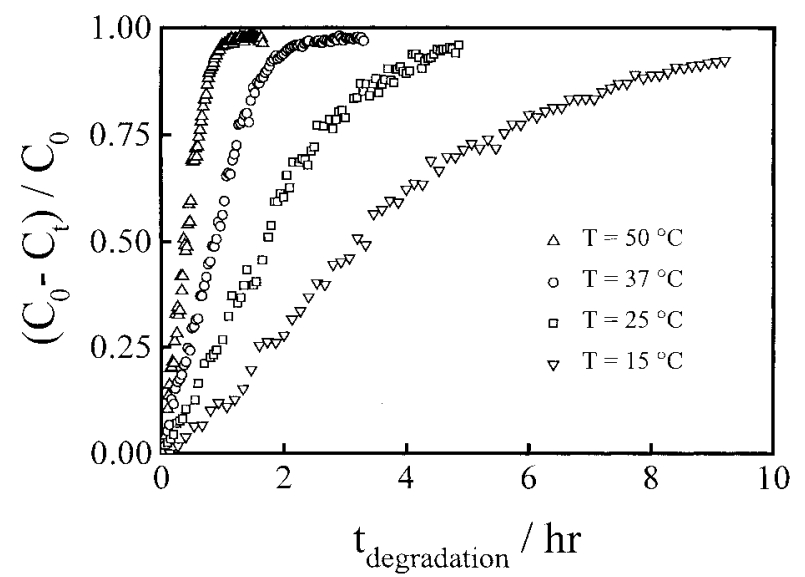

Figure 7. Temperature dependence of the degradation of SDS-PSA nanoparticles, where $C_{o}$ is $3.88 \times 10^{-6}$ $\mathrm{g} / \mathrm{mL}$ and the $\mathrm{pH}$ is 10.0 .

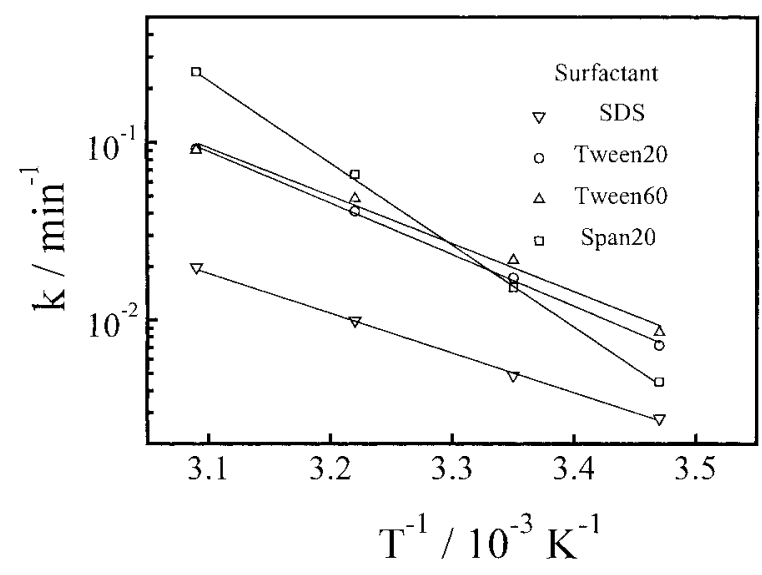

Figure 8. Arrhenius plots of the rate constant $k$ versus the reciprocal absolute temperature $T^{-1}$ for the degradation of PSA nanoparticles in a buffer solution.

degradation faster but also leads to a zero-order degradation kinetics up to a $75 \%$ weight loss, which can be attributed to the fast degradation of the PSA nanoparticles and a constant decreasing rate of the nanoparticle number during the degradation. In this way, the constant total surface required for the zero-order kinetics can be relaxed. The degradation rate of the PSA nanoparticles increases as $\mathrm{pH}$ and temperature increase. The activation energy of the degradation ranges from 40 to $90 \mathrm{~kJ} / \mathrm{mol}$. Our results also show that surfactant used as a stabilizer in the micronization of PSA can affect the degradation rate. Generally, an anionic surfactant or a surfactant with a polyoxyethylene chains leads to a slower degradation.

The financial support of the Research Grants Council of the Hong Kong Special Administration Region (Earmarked Grants 1999/2000: CUHK4209/99P, 2160122), the CAS Bai Ren Project, and the NNSFC project (29974027) is gratefully acknowledged. J. Fu is grateful to the Chinese University of Hong Kong for partially supporting her 1-year postdoctoral.

\section{REFERENCES AND NOTES}

1. Peppas, N.; Langer, R. Science 1994, 263, 1657.

2. Langer, R. Science 1990, 249, 1527.

3. Langer, R.; Vacanti, J. Science 1993, 260, 920.

4. Chandra, R.; Rustgi, R. Prog Polym Sci 1998, 23, 1273.

5. Davis, S. S. J Pharm Pharmacol 1992, S1, 186.

6. Heller, J. Adv Drug Delivery Rev 1993, 10, 163.

7. Tamada, J.; Langer, R. J Biomater Sci Polym Ed 1992, 3, 315 .

8. Gopferich, A. Biomaterials 1996, 17, 103. 
9. Mathiowitz, E.; Amato, C.; Dor, P.; Langer, R. Polymer 1990, 31, 547.

10. Mathiowitz, E.; Saltzman, W.; Domb, A.; Dor, P.; Langer, R. J Appl Polym Sci 1988, 35, 755.

11. Mathiowitz, E.; Bernstein, H.; Giannos, S.; Dor, P.; Turek, T.; Langer, R. J Appl Polym Sci 1992, 45, 125.

12. Ron, E.; Turek, T.; Mathiowitz, E.; Chasin, M.; Hageman, M.; Langer, R. Proc Natl Acad Sci USA 1993, 90, 4167.

13. Leong, K. W.; Brott, B. C.; Langer, R. J Biomed Mater Res 1985, 19, 941.

14. Laurencin, C.; Domb, A.; Morris, C.; Brown, V.; Chasin, M.; McConnell, R.; Lange, N.; Langer, R. J Biomed Mater Res 1990, 24, 1463.

15. Brem, H.; Piantadosi, S.; Burger, P. C.; Walker, M.; Selker, R.; Vick, N. A.; Black, K.; Sisti, M.; Brem, S.; Mohr, G.; Muller, P.; Morawetz, R.; Schold, S. C. Lancet 1995, 345, 1008.

16. Kreuter, J. M. In Microcapsules and Nanoparticles in Medicine and Pharmacy; Donbrow, M., Ed.; CRC: Boca Raton, FL, 1992; pp 126-143.

17. Allemann, E.; Doelker, E.; Gurny, R. J Pharm Biopharm 1993, 39, 13.

18. Fessi, H.; Puiosieux, F.; Devissaguet, J. P.; Benita, S. Int J Pharm 1989, 55, R1.

19. Scholas, P. D.; Coombes, A. G.; Illum, L.; Davis, S. S.; Vert, M.; Davis, M. C. J Controlled Release 1993, 25, 145

20. Molpeceres, J.; Guzman, M.; Aberturas, M. R.; Chacon, M.; Berges, L. J Pharm Sci 1996, 85, 206.
21. Chacon, M.; Berges, L.; Molpereres, J.; Aberturas, M. R.; Guzman, M. Int J Pharm 1996, 141, 81.

22. Lemoine, D.; Francois, C.; Kedzierwicz, F.; Preat, V.; Hoffman, M.; Maincent, P. Biomaterials 1996, 17, 2191.

23. Kreuter, J. J Controlled Release 1991, 16, 169.

24. Maincent, P.; Le Verge, R.; Sado, P.; Couvreur, P.; Devissaguet, J. P. J Pharm Sci 1986, 75, 955.

25. Couvreur, P.; Grislain, L.; Lenaerts, V.; Brasseur, P.; Guiot, P. In Polymeric Nanoparticles and Microspheres; Guiot, P.; Couvreur, P., Eds.; CRC: Boca Raton, FL, 1986; pp 27-93.

26. Li, M.; Jiang, M.; Zhu, L.; Wu, C. Macromolecules 1997, 30, 2201.

27. Wu, C.; Akashi, M.; Chen, M. Macromolecules 1997, 30, 2187.

28. Gan, Z.; Jim, T. F.; Li, M.; Zhao, Y.; Wang, S. G.; Wu, C. Macromolecules 1999, 32, 5901.

29. Domb, A. J.; Langer, R. J Polym Sci Part A: Polym Chem 1987, 25, 3373.

30. Domb, A.; Mathiowitz, E.; Giannos, S.; Langer, R. J Polym Sci Part A: Polym Chem 1991, 29, 571.

31. Domb, A.; Gallardo, C.; Langer, R. Macromolecules 1989, 22, 3200.

32. Chu, B. Laser Light Scattering, 2nd ed.; Academic: New York, 1991, 94.

33. Berne, B.; Pecora, R. Dynamic Light Scattering; Plenum: New York, 1976, 33.

34. Wu, C.; Zuo, J.; Chu, B. Macromolecules 1989, 22, 633. 